\title{
EDITORIAL
}

\section{Making Integrated Multimodal Pain Care a Reality: A Path Forward}

\author{
Robert D. Kerns, $P h D^{7}$, Erin E. Krebs, $M D, M P H^{2}$, and David Atkins, $M D, M^{2} H^{3}$ \\ 'Departments of Psychiatry, Neurology and Psychology, Yale University, New Haven, CT, USA; ${ }^{2}$ University of Minnesota Medical School, \\ Minneapolis, MN, USA; ${ }^{3}$ Office of Research and Development, Department of Veterans Affairs (10P9H), Washington DC, USA.
}

J Gen Intern Med 33(Suppl 1):S1-S3

DOI: $10.1007 / \mathrm{s} 11606-018-4361-6$

(c) Society of General Internal Medicine 2018

I n the fall of 2015, the White House convened a national summit involving multiple federal agencies to address the national epidemic of opioid use. At that summit, the Secretary for Veterans Affairs (VA) Robert MacDonald volunteered the VA to take the lead in examining the role of complementary and integrative health $(\mathrm{CIH})$ approaches as a way to reduce over-use of opioids in chronic pain management. As the largest integrated healthcare system in the USA, VA serves a patient population heavily affected by chronic pain. ${ }^{1}$ Since 2000 , over five million veterans receiving care in VA were diagnosed with chronic musculoskeletal pain. ${ }^{2}$ VA was an early leader in developing a comprehensive national pain management strategy ${ }^{3}$ and then an initiative that significantly reduced high-risk opioid prescribing (i.e., Opioid Safety Initiative). Moreover, VA has provided chiropractic care for more than 10 years and has more recently expanded $\mathrm{CIH}$ offerings such as acupuncture, yoga, and meditation.

VA activities following the summit included a systematic review on the effects of $\mathrm{CIH}$ on pain and opioid use, an expert planning meeting to frame a larger discussion about approaches to chronic pain, and a State-Of-The-Art Conference on the broader topic of non-opioid therapies in November 2016. The SOTA planners expanded the focus from $\mathrm{CIH}$ to non-pharmacological approaches, including structured exercise and cognitive behavioral therapy (CBT), and narrowed the focus on chronic musculoskeletal pain, the most common target of opioid therapy. The conference included researchers, clinical experts, and other stakeholders from the VA, Department of Defense, National Institutes of Health, and the academic community. Three work groups were formed to focus on psychological/behavioral, exercise/movement, and manual approaches. A fourth work group examined evidence supporting different models of pain care delivered in general medical settings. Goals of the conference were to issue recommendations for VA clinical practice-where supported by existing evidence - and recommendations for research to address important gaps in the evidence.

Published online April 9, 2018
This SOTA built on previous national efforts targeting chronic pain. In its 2011 report, "Relieving Pain in America," the Institute of Medicine (IOM) asserted pain is a major public health problem, with 100 million Americans affected at a cost of $\$ 635$ billion. ${ }^{4}$ Following the recommendations of this report, the Department of Health and Human Services (HHS) published a National Pain Strategy (NPS) that offered a comprehensive framework for transforming pain care in America. ${ }^{5}$ The NPS recommended that pain care should be multidisciplinary, multimodal, and tailored to individuals. Although the VA has made important progress in adopting the recommendations in the NPS, the SOTA was designed to consider more recent evidence, especially regarding $\mathrm{CIH}$, and identify where VA still had room to improve.

\section{CLINICAL RECOMMENDATIONS}

SOTA participants were asked to use existing reviews, contextual evidence, and their own expertise to distill and translate evidence into recommendations. This resulted in recommendations for broad implementation of specific psychological/ behavioral, exercise/movement, and manual therapies supported by sufficient evidence, as detailed in this issue by Kligler and colleagues. ${ }^{6}$

These clinical recommendations overlap substantially with those of existing clinical practice guidelines for conditions such as back pain and osteoarthritis. Unfortunately, recommended evidence-based therapies are often set aside in favor of riskier and less proven approaches. Most recommended non-pharmacologic therapies are not widely available in health care settings, are often excluded from insurance coverage, and - for many patients - provided only after opioids and interventional procedures fail.

A problem in focusing on "alternatives" to opioids is that it gives undue weight to the scant evidence that opioids are effective in chronic pain. Instead, we think long-term opioid therapy should be regarded as the unproven "alternative" to evidence-based non-pharmacologic therapies, which are supported by stronger evidence than that for opioids, including evidence from trials with long-term pain and functional outcomes.

Nearly all non-pharmacological therapies are safer than medications and invasive procedures, but not all are ready 
for broad implementation. In recommending specific approaches, SOTA workgroups also decided not to recommend others. Given the number of existing therapies with established evidence, we should not use the opioid crisis as an excuse to bypass scientific standards of what treatments to adopt widely in VA.

Evidence clearly shows that no single therapy is the best approach for a majority of patients with chronic musculoskeletal pain. Like analgesic medications, non-pharmacologic therapies generate meaningful clinical improvement in only a subset of patients. This has three important implications for health systems and payers. First, multiple options should be available to patients. As health systems consider how to adopt complementary therapies such as yoga and mindfulness-based stress reduction, they should also ensure access to wellestablished approaches that receive less media attention (e.g., structured exercise, CBT). Second, combining therapies (i.e., multimodal care) will often be necessary to achieve greater treatment effects for individual patients. And third, systematic support for structured trial-and-error, as described in Peterson et al.'s ${ }^{7}$ review of approaches to delivering multimodal care, is needed to efficiently and effectively manage pain care.

Patients usually have to devote considerable effort to obtain meaningful benefits from psychological/behavioral and exercise/movement therapies. Health systems and payers, therefore, need to support sufficient "dosing" and maintenance of behavior change. For example, effective exercise therapy programs include individual tailoring, therapist supervision, and more treatment sessions. Limited or brief programs are unlikely to generate similar benefits. Even worse, repeated sub-therapeutic exposures to active therapies (e.g., three physical therapy visits in lieu of a robust exercise therapy program) could decrease patients' future willingness to engage - what Kurt Kroenke has described as "immunizing" them against a potentially beneficial therapy.

Opportunities nonetheless exist to successfully implement the therapies recommended from the SOTA, especially within primary care. Primary care teams are well suited to deliver optimal pain care, which typically involves long-term conservative multimodal management of patients with common, readily-diagnosed chronic conditions. Further, risk factors for chronic pain-including behavioral factors and social determinants - overlap with risk factors for important chronic conditions commonly managed in primary care, including diabetes and depression, and these chronic conditions frequently coexist. ${ }^{8}$ Many of the SOTA-recommended interventions (e.g., exercise therapies, CBT, MBSR) are either standard or emerging treatments for chronic conditions such as depression, making it especially important to expand access to them.

\section{RESEARCH RECOMMENDATIONS}

SOTA recommendations for research are outlined in the paper by Becker and colleagues. ${ }^{9}$ As the Office of Research and
Development considers these recommendations, we will focus on questions that seem most critical for making effective integrated, multimodal pain care a reality across the VA. The critical questions are no longer about effectiveness of individual therapies or superiority of one over another. As noted by the SOTA, and echoed by a recent AHRQ review, ${ }^{10}$ a variety of non-opioid strategies have benefit, but clinical effects of any individual approach are modest on average and individual responses vary substantially.

Two lines of research could help make integrated, multimodal stepped models of care a reality in VA. The first is to test how to make individual therapies more effective, affordable, and scalable. For manual therapies requiring inperson visits, we need to know more about the frequency and duration of therapy necessary to sustain benefits. For exercise/movement therapies, we need to test approaches such as group sessions and on-line coaching. Finally, for psychological/behavioral therapies, we need to examine effectiveness of delivery through telehealth or internet modes. Answers to these questions will determine how many patients we can serve, how convenient it will be for patients, and what it will cost VA to do this throughout a diverse, national system.

The second critical line of research is how to structure and implement stepped care models in primary care. Some of this research is already underway - a large trial funded by the Patient Centered Outcomes Research Institute (PCORI) led by one of us (Krebs) will compare two different primary care-based collaborative models in nine sites across the VA. This study will compare the models' effectiveness and also yield insights into implementation challenges for each model. Making real progress in the care of patients with pain, and reducing reliance on opioids for treatment of such patients, requires getting these models implemented beyond the few centers that have pioneered them or studied them in funded research. It will require developing the training curricula, information technology tools, budget analyses, and facilitation support to help new sites implement new ways of taking care of some of their most challenging patients. Finally, we need more effective ways of monitoring individual responses to therapies so that new therapies can be offered when the existing one is not working.

The challenges of bringing the wealth of effective nonopioid therapies and integrated programs to scale in a national system are daunting. As with individual therapies, there is no "silver bullet". The good news is that there is growing scientific and clinical consensus that primary care clinicians have new weapons in the fight against chronic pain, weapons that carry fewer risks and are probably more effective in the long term than opioids. Our responsibility is to support the implementation of what we know, even as we develop better evidence about what we do not yet know. 
Corresponding Author: David Atkins, MD, MPH; Office of Research and Development, Department of Veterans Affairs (10P9H), 810 Vermont Ave NW, Washington DC 20420, USA (e-mail: david. atkins@va.gov).

\section{Compliance with Ethical Standards:}

Conflict of Interest: The authors declare that they do not have a conflict of interest.

\section{REFERENCES}

1. Lew HL, Otis JD, Tun C, Kerns RD, Clark ME, Cifu DX. Prevalence of chronic pain, posttraumatic stress disorder, and post-concussive syndrome in OEF/OIF veterans: The polytrauma clinical triad. J Rehab Res Develop 2009; 46: 697-702.

2. Goulet JL, Kerns RD, Bair M, et al. The Musculoskeletal Diagnosis Cohort: Examining pain and pain care among veterans. Pain 2016; 157: 1696-1703.

3. Department of Veterans Affairs. VHA National Pain Management Strategy. Accessed from https://www.va.gov/PAINMANAGEMENT/VHA Pain_Management_Strategy.asp on December 10, 2017.
4. Institute of Medicine. Relieving pain in America: A blueprint for transforming pain prevention, care, education and research. Washington, D.C.: The National Academies Press, 2011.

5. Department of Health and Human Services. National Pain Strategy: A comprehensive population health-level strategy for pain. Accessed from https://iprcc.nih.gov/sites/default/files/HHSNational_Pain_Strategy_ 508C.pdf on December 10, 2017.

6. Kligler B, et al. Clinical Policy Recommendations form the VHA State of the Art Conference on Non-Pharmalogical Approaches to Chronic Musculoskeletal Pain. J Gen Intern Med. https://doi.org/10.1007/ s11606-018-4323-Z.

7. Peterson K, et al. Effectiveness of Models Used to Deliver Multimodal Care for Chronic Musculoskeletal Pain: A Rapid Evidence Review. J Gen Intern Med. https://doi.org/10.1007/s11606-018-4328-7.

8. Bair MJ, Wu J, Damush TM, Sutherland JM, Kroenke K. Association of depression and anxiety alone and in combination with chronic musculoskeletal pain in primary care patients. Psychosomatic Med 2008; 70: 890-897.

9. Becker WC, et al. A Research Agenda for Advancing NonPharmacological Management of Chronic Musculoskeletal Pain: Findings from a VHA state of the art conference. J Gen Intern Med. https://doi. org/10.1007/s11606-018-4345-6.

10. Agency for Healthcare Research and Quality: Noninvasive, nonpharmacological treatment for chronic pain: a systematic review (protocol). Accessed from https://www.effectivehealthcare.ahrq.gov/topics/ nonpharmatreatment-pain/research-protocol on February 21, 2018. 